\title{
BMJ
}

\section{Effect of reduced immunosuppression after kidney transplant failure on risk of cancer: population based retrospective cohort study}

\begin{abstract}
Marina T van Leeuwen, lecturer, epidemiologist, ${ }^{1,2}$ Angela C Webster, senior lecturer, medical epidemiologist, and nephrologist, ${ }^{3,4,5}$ Margaret R E McCredie, associate professor, epidemiologist, ${ }^{6}$ John H Stewart, nephrologist, ${ }^{6}$ Stephen P McDonald, associate professor, nephrologist, ${ }^{3,7}$ Janaki Amin, senior lecturer, statistician, ${ }^{1}$ John M Kaldor, professor, epidemiologist, ${ }^{1}$ Jeremy R Chapman, professor, nephrologist, ${ }^{5}$ Claire M Vajdic, senior lecturer, epidemiologist, ${ }^{8}$ Andrew E Grulich, professor, medical epidemiologist ${ }^{1}$
\end{abstract}

${ }^{1}$ National Centre in HIV

Epidemiology and Clinical

Research, University of New South

Wales, 376 Victoria St, Sydney,

NSW, 2010, Australia

${ }^{2}$ School of Public Health and Community Medicine, University of New South Wales, Sydney, NSW, 2052, Australia

${ }^{3}$ Australia and New Zealand Dialysis and Transplant Registry, Queen Elizabeth Hospital,

Woodville South, SA, 5011, Australia

${ }^{4}$ School of Public Health, University of Sydney, Sydney, NSW, 2006, Australia

${ }^{5}$ Centre for Transplant and Renal Research, Westmead Millennium Institute, University of Sydney, Westmead Hospital, Westmead, NSW, 2145, Australia

${ }^{6}$ Department of Preventive and Social Medicine, University of Otago, Dunedin, 9054, New Zealand

${ }^{7}$ Disciplines of Medicine and Public Health, University of Adelaide, Adelaide, SA, 5005, Australia

${ }^{8}$ University of New South Wales Cancer Research Centre, Prince of Wales Clinical School, University of New South Wales, Sydney, NSW, 2052, Australia Correspondence to: A E Grulich agrulich@nchecr.unsw.edu.au

Cite this as: $B M J$ 2010;340:с570 doi:10.1136/bmj.c570

\section{ABSTRACT}

Objective To compare cancer incidence in kidney transplant recipients during periods of transplant function (and immunosuppression) and after transplant failure (when immunosuppression is ceased or reduced) Design, setting, and participants Nationwide, population based retrospective cohort study of 8173 Australian kidney transplant recipients registered on the Australia and New Zealand Dialysis and Transplant Registry who first received a transplant during 1982-2003. Incident cancers were ascertained using linkage with national cancer registry records.

Main outcome measures Cancer-specific standardised incidence ratios for periods of transplant function and for dialysis after transplant failure. Incidence was compared between periods using multivariate incidence rate ratios adjusted for current age, sex, and duration of transplantation.

Results All cases of Kaposi's sarcoma occurred during transplant function. Standardised incidence ratios were significantly elevated during transplant function, but not during dialysis after transplant failure, for non-Hodgkin's lymphoma, lip cancer, and melanoma. For each of these cancers, incidence was significantly lower during dialysis after transplant failure in multivariate analysis (incidence rate ratios $0.20(95 \% \mathrm{Cl} 0.06$ to 0.65$)$ for non-Hodgkin's lymphoma, 0.04 (0.01 to 0.31 ) for lip cancer, and 0.16 (0.04 to 0.64 ) for melanoma). In contrast, standardised incidence ratios during dialysis after transplant failure remained significantly elevated for leukaemia and lung cancer, and cancers related to end stage kidney disease (kidney, urinary tract, and thyroid cancers), with thyroid cancer incidence significantly higher during dialysis after transplant failure (incidence rate ratio 6.77 (2.64 to 17.39)). There was no significant difference in incidence by transplant function for other cancers.

Conclusions The effect of immunosuppression on cancer risk is rapidly reversible for some, but not all, cancer types. Risk reversal was mainly observed for cancers with a confirmed infectious cause. Risk of other cancers, especially those related to end stage kidney disease, remained significantly increased after reduction of immunosuppression.

\section{INTRODUCTION}

A broad range of cancers, particularly those related to infection, occur at excess rates after solid organ transplantation - a situation similar to that seen in HIV infection. ${ }^{1}$ In HIV infection, the partial reversal of immunodeficiency that is associated with antiretroviral therapy rapidly and substantially reduces the risk of Kaposi's sarcoma ${ }^{2}$ and non-Hodgkin's lymphoma. ${ }^{3}$ There are few data on whether the risk of other cancers is also reduced. ${ }^{4-6}$ In transplant recipients, clinical regression of cases of Kaposi's sarcoma ${ }^{78}$ and non-Hodgkin's lymphoma ${ }^{910}$ has been described after reduction of iatrogenic immunosuppression. However, as in HIV infection, there are few data on whether cancer risk is reduced when immunosuppression is ceased.

In kidney transplant recipients, transplant failure generally leads to the reinstitution of dialysis and the substantial reduction or complete withdrawal of immunosuppression. ${ }^{11}$ This offers a unique opportunity to examine the cancer risk profile on reduction of immunosuppression. ${ }^{12}$ Recently, we showed that incidence of lip cancer, ${ }^{13}$ non-Hodgkin's lymphoma, ${ }^{14}$ and melanoma $^{15}$ decreased markedly after kidney transplant failure and reinstitution of dialysis. In the present paper, we report on the site-specific pattern of cancer occurrence during periods of transplant function and during periods of dialysis after transplant failure in an Australian cohort of kidney transplant recipients.

\section{METHODS}

Data sources

The Australia and New Zealand Dialysis and Transplant (ANZDATA) Registry is a comprehensive, population based registry of all patients commencing maintenance dialysis or undergoing kidney 
transplantation in Australia and New Zealand. ${ }^{16}$ Our study cohort included all patients on the ANZDATA Registry in Australia who first received a transplant between 1 January 1982 and 30 September 2003 $(\mathrm{n}=8173)$. Incident cancers were ascertained through probabilistic record linkage with the Australian National Cancer Statistics Clearing House, which contains data on all diagnoses of incident invasive cancer in Australia since 1982, with the exception of non-melanoma skin cancer. ${ }^{17}$

For each matched record, we obtained the date of cancer diagnosis and ICDO-3 and ICD-10 (international classifications of diseases) codes for topography and morphology. ${ }^{18}$ We also obtained the general population's cancer incidence rates by five year age group, sex, calendar year, and state or territory, for each year since 1982. Data were available to 2001, 2002 , or 2003 depending on jurisdiction of cancer registration.

\section{Statistical analysis}

Person years of follow-up were accumulated from the date of first transplantation until the date of death, last contact, the latest date for which cancer data were available, or the first diagnosis of each type of cancer. Cancer incidence was examined during periods of transplant function and of dialysis after transplant failure. Person years and incident cancers during the first three months of each period were excluded, as cancers diagnosed within this time would almost certainly have developed in the preceding period.

For each period, we calculated cancer-specific standardised incidence ratios (with 95\% confidence intervals) by comparing the number of observed cases with that expected based on the application of the appropriate population cancer incidence rates. ${ }^{19}$ The exception was for Kaposi's sarcoma, in which we applied population rates from 1982 because of the impact of AIDS related Kaposi's sarcoma in the years thereafter.

Comparison of period-specific standardised incidence ratios may be confounded by differences in the age and sex structure of the cohort over time. Therefore, for each cancer, we used multivariate Poisson regression to compute incidence rate ratios with 95\% confidence intervals comparing incidence during each period, with adjustment for current age (time dependent, single years), sex, and the cumulative duration of transplant function (time dependent, years). For cancers with zero cases during dialysis, we calculated a median unbiased estimate of the incidence rate ratio using exact Poisson regression adjusted for current age (time dependent, $<35,35-50, \geq 50$ years), sex, and duration of transplant function (time dependent, $<2,2$ $4.99,5-9.99,10-14.99, \geq 15$ years) ${ }^{20}$

A two sided $\mathrm{P}$ value of $<0.05$ was considered statistically significant. All analyses were performed using Stata version 10 (StataCorp, College Station, TX, USA).

\section{Grouping of cancers}

Analyses were restricted to cancers or groups of cancers (anogenital, oral cavity, and oropharyngeal cancers) for which there were at least 10 cases in total. For descriptive purposes, cancers were grouped according to their association with infection, end stage kidney disease, or immunodeficiency. Cancers associated with infection were those for which there is sufficient evidence of causality according to the International Agency for Research on Cancer. ${ }^{21}$ These included Kaposi's sarcoma (human herpesvirus type 8); immunosuppression related non-Hodgkin's lymphoma and Hodgkin's lymphoma (Epstein-Barr virus); anogenital, oropharyngeal, and oral cavity cancers (human papillomavirus); liver cancer (hepatitis B virus and hepatitis $\mathrm{C}$ virus); and cancer of the stomach (Helicobacter pylori).

Cancers associated with end stage kidney disease (cancers of the kidney, urinary tract, and thyroid) were those for which our group had previously shown the relative risk to be at least doubled before renal replacement therapy, ${ }^{18}$ and for which there is no, or a much smaller, excess risk in people infected with HIV. ${ }^{1}$ Remaining cancers were grouped according to their association with immunodeficiency, based on whether they occur at increased rates after both solid organ transplantation and HIV infection according to meta-data. ${ }^{1}$

\section{RESULTS}

The study cohort comprised 7809 patients (4628 males, 3181 females), 364 patients having been censored because of exclusion of follow-up during the first three months of each period. In total, 925 cancers were identified; $33(3.4 \%)$ of these were excluded because they occurred in the first three months of the transplant $(\mathrm{n}=11)$ or dialysis $(\mathrm{n}=22)$ period. This left 892 cancers identified over 59037 person years. The median age of patients at first transplantation was 43 years. Failure of the first transplant necessitating institution of dialysis occurred in $1820(23 \%)$ patients, and $668(9 \%)$ underwent a second transplantation. Three or more transplants were received by $66(1 \%)$ patients. In total, there were 51933 person years (mean 6.9) during periods of transplantation and 7104 person years (mean 3.9) during dialysis after transplant failure.

\section{Incidence of cancer by transplant function}

The figure shows the cancer-specific standardised incidence ratios for the periods of transplant function and dialysis after transplant failure standardised by five year age group, sex, state or territory, and calendar year. It also shows the multivariate incidence rate ratios comparing incidence during dialysis after transplant failure with that during transplant function, adjusted for current age, sex, and cumulative duration of transplant function. 


\begin{tabular}{|c|c|c|c|c|c|c|c|c|}
\hline \multirow{2}{*}{$\begin{array}{l}\text { Cancer site* } \\
\text { Infection related }\end{array}$} & \multirow[t]{2}{*}{$\begin{array}{l}\text { Transplant } \\
\text { function }\end{array}$} & \multirow[t]{2}{*}{$\begin{array}{c}\text { SIR } \\
(95 \% \mathrm{Cl})\end{array}$} & \multicolumn{3}{|c|}{$\begin{array}{c}\text { SIR } \\
(95 \% \mathrm{Cl})\end{array}$} & \multirow{3}{*}{$\begin{array}{c}\begin{array}{c}\text { No of } \\
\text { cases }\end{array} \\
10\end{array}$} & \multirow{2}{*}{$\begin{array}{c}\text { IRR } \\
(95 \% \mathrm{CI})\end{array}$} & \multirow[t]{2}{*}{ P value } \\
\hline & & & & & & & & \\
\hline \multirow[t]{2}{*}{ Kaposi's sarcoma } & Transplant & 231 (111 to 425) & & & $\rightarrow-$ & & 1.00 & \\
\hline & Dialysis & Upper Cl 622† & & & & 0 & 0.25 (0.00 to 1.65$) \ddagger$ & 0.175 \\
\hline \multirow[t]{2}{*}{ Non-Hodgkin's lymphoma } & Transplant & 9.73 (8.08 to 11.62$)$ & & . & & 122 & 1.00 & \\
\hline & Dialysis & 2.05 (0.42 to 5.99$)$ & & $\rightarrow \cdots$ & & 3 & 0.20 (0.06 to 0.65$)$ & 0.007 \\
\hline \multirow[t]{2}{*}{ Anogenital } & Transplant & 5.96 (3.93 to 8.67$)$ & & 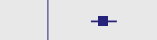 & & 27 & 1.00 & \\
\hline & Dialysis & $1.82(0.05$ to 10.11$)$ & & $\bullet$ & & 1 & 0.41 (0.05 to 3.04$)$ & 0.380 \\
\hline \multirow[t]{2}{*}{ Oral cavity and oropharynx } & Transplant & $3.46(2.01$ to 5.53$)$ & & $\rightarrow$ & & 17 & 1.00 & \\
\hline & Dialysis & Upper $\mathrm{Cl} 6.32 †$ & & ---- & & 0 & $0.36(0.00$ to 2.21$) \neq$ & 0.322 \\
\hline \multirow[t]{2}{*}{ Stomach } & Transplant & $1.76(0.85$ to 3.24$)$ & & - & & 10 & 1.00 & \\
\hline & Dialysis & 2.89 (0.35 to 10.45$)$ & & 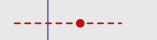 & & 2 & $1.46(0.30$ to 7.16$)$ & 0.640 \\
\hline \multicolumn{9}{|c|}{ Increased in immunodeficient populations } \\
\hline \multirow[t]{2}{*}{ Lip } & Transplant & 52.27 (45.27 to 60.02$)$ & & & - & 200 & 1.00 & \\
\hline & Dialysis & $2.16(0.05$ to 12.05$)$ & & $-\bullet----$ & & 1 & 0.04 (0.01 to 0.31$)$ & 0.002 \\
\hline \multirow[t]{2}{*}{ Melanoma } & Transplant & $2.74(2.17$ to 3.41$)$ & & $=$ & & 80 & 1.00 & \\
\hline & Dialysis & 0.58 (0.07 to 2.08$)$ & & 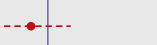 & & 2 & $0.16(0.04$ to 0.64$)$ & 0.010 \\
\hline \multirow[t]{2}{*}{ Leukaemia } & Transplant & 2.58 (1.38 to 4.42$)$ & & $\rightarrow$ & & 13 & 1.00 & \\
\hline & Dialysis & 5.25 (1.08 to 15.33$)$ & & $\cdots \cdots$ & & 3 & $1.52(0.41$ to 5.67$)$ & 0.533 \\
\hline \multirow[t]{2}{*}{ Lung } & Transplant & 2.14 (1.61 to 2.77$)$ & & $=$ & & 56 & 1.00 & \\
\hline & Dialysis & $2.59(1.12$ to 5.11$)$ & & $-\cdots \cdot-$ & & 8 & 1.24 (0.58 to 2.68$)$ & 0.579 \\
\hline \multicolumn{9}{|c|}{ Not increased in immunodeficient populations } \\
\hline \multirow[t]{2}{*}{ Colon } & Transplant & 1.75 (1.24 to 2.39$)$ & & $\rightarrow$ & & 39 & 1.00 & \\
\hline & Dialysis & $1.99(0.65$ to 4.65$)$ & & $-\cdots$ & & 5 & $1.32(0.50$ to 3.46$)$ & 0.571 \\
\hline \multirow[t]{2}{*}{ Breast (female) } & Transplant & $0.97(0.66$ to 1.36$)$ & & + & & 33 & 1.00 & \\
\hline & Dialysis & 0.54 (0.06 to 1.93$)$ & & $\ldots$ & & 2 & 0.57 (0.13 to 2.42$)$ & 0.445 \\
\hline \multirow[t]{2}{*}{ Prostate } & Transplant & 0.70 (0.44 to 1.06$)$ & & - & & 22 & 1.00 & \\
\hline & Dialysis & 1.05 (0.29 to 2.69$)$ & & $\rightarrow$ & & 4 & $1.56(0.52$ to 4.73$)$ & 0.430 \\
\hline \multicolumn{9}{|c|}{ End stage kidney disease related } \\
\hline \multirow[t]{2}{*}{ Kidney } & Transplant & $4.93(3.35$ to 7.00$)$ & & + & & 31 & 1.00 & \\
\hline & Dialysis & 12.38 (5.66 to 23.49$)$ & & $--\bullet$ & & 9 & 2.08 (0.96 to 4.51$)$ & 0.064 \\
\hline \multirow[t]{2}{*}{ Urinary tract } & Transplant & $3.69(2.45$ to 5.33$)$ & & $\rightarrow$ & & 28 & 1.00 & \\
\hline & Dialysis & 7.07 (2.59 to 15.38$)$ & & $-\cdots--$ & & 6 & $1.77(0.70$ to 4.44$)$ & 0.225 \\
\hline \multirow[t]{3}{*}{ Thyroid } & Transplant & $3.29(1.58$ to 6.05$)$ & & $\rightarrow-$ & & 10 & 1.00 & \\
\hline & Dialysis & 26.37 (12.64 to 48.49$)$ & & $--\bullet$ & & 10 & 6.77 (2.64 to 17.39$)$ & $<0.001$ \\
\hline & & & 0.1 & 10 & 100 & 1000 & & \\
\hline
\end{tabular}

Cancer-specific standardised incidence ratios (SIR) by transplant function and multivariate incidence rate ratios (IRR). *ICDO-3 and ICD-10 codes for cancers: Kaposi's sarcoma (C46); non-Hodgkin's lymphoma (ICDO-3 9591, 9670-9729, 9820-9837, 9940, 9948, and 9590 if ICD-10 C82-C85); anogenital (C21 anus, C51-53 vulva, vagina, cervix uteri, C60 penis); oropharyngeal and oral cavity (C01-C02 tongue, C03-C06 mouth, C09 tonsil, C10 oropharynx); stomach (C16); lip (C00); melanoma (C43); leukaemia (ICD0-3 9800-9989 excluding 9820-9837, 9940, and 9948); lung (C33-34); colon (C18); breast (C50); prostate (C61); kidney (C64); urinary tract (C65-C68); thyroid (C73). †Upper confidence interval presented when zero cases observed. $\ddagger$ Median unbiased estimate.

\section{Cancers related to infection}

The standardised incidence ratio for Kaposi's sarcoma (10 cases) was substantially elevated during transplant function; multivariate analysis was not performed as no cases occurred during dialysis after transplant failure.

The standardised incidence ratio for non-Hodgkin's lymphoma was significantly elevated during transplant function, but not during dialysis after transplant failure. In the multivariate analysis with adjustment for current year of age, sex, and the cumulative duration of transplant function, cancer incidence was significantly lower during dialysis after transplant failure than during transplant function.

Standardised incidence ratios for cancers associated with human papillomavirus infection (anogenital, oral cavity, and oropharyngeal cancers) were significantly elevated during transplant function, but not during dialysis after transplant failure. In multivariate analysis, incidence was not significantly lower during dialysis after transplant failure, including when all the cancer sites associated with human papillomavirus were combined (incidence rate ratio 0.24 (95\% confidence interval 0.03 to 1.74$), \mathrm{P}=0.157)$.

Standardised incidence ratios for stomach cancer were not significantly elevated, and there was no difference in incidence between periods in multivariate analysis.

There were insufficient cases of other infection related cancers, including Hodgkin's lymphoma $(n=7)$ and liver cancer $(n=4)$, for analysis. 
Cancers related to end stage kidney disease

For cancers of the thyroid, kidney, and urinary tract, standardised incidence ratios were significantly elevated both during transplant function and dialysis after transplant failure. In multivariate analysis, incidence was significantly increased during dialysis after transplant failure for thyroid cancer and was non-significantly increased for cancer of the kidney. There was no difference in incidence between periods for cancers of the urinary tract.

\section{Other cancers}

Standardised incidence ratios were significantly elevated during transplant function, but not during subsequent dialysis, for lip cancer and melanoma. For both cancers, incidence was significantly lower during dialysis after transplant failure in multivariate analysis. Standardised incidence ratios for leukaemia and lung cancer were significantly elevated during both transplant function and dialysis after transplant failure, and there was no difference in incidence between periods in multivariate analysis. The standardised incidence ratio for colon cancer was slightly elevated during transplant function but not during dialysis after transplant failure, and there was no difference in incidence between periods in multivariate analysis. Standardised incidence ratios were not elevated in either period for cancers of the breast or prostate, and there was no difference in incidence between periods in multivariate analysis.

\section{DISCUSSION}

Among those cancers known to occur at increased rates in kidney transplant recipients, the pattern of incidence during dialysis after transplant failure was highly variable. The incidence of Kaposi's sarcoma and nonHodgkin's lymphoma (the infection related cancers most strongly related to immunosuppression in both transplant recipients and people infected with HIV) decreased markedly on reinstitution of dialysis. The incidence of lip cancer and melanoma also declined substantially. For each of these four cancer types, standardised incidence ratios were significantly elevated during periods of transplant function, but not during dialysis after transplant failure, suggesting that the effect of immunosuppression on cancer risk was close to completely reversible.

Standardised incidence ratios remained elevated during dialysis after transplant failure for leukaemia and lung cancer, and for cancers known to be related to end stage kidney disease (urinary tract, kidney, thyroid). In fact, the incidence of thyroid cancer was significantly higher during dialysis after transplant failure than during transplant function. There was no significant variation in incidence for other cancers.

\section{Strengths and limitations of the study}

This study had several strengths, including the use of national, population based registers of cancer and kidney transplantation, the long period of follow-up, and, unique to the ANZDATA Registry, the availability of comprehensive data on consecutive periods of transplantation and dialysis. The principal limitation was limited statistical power with which to examine incidence beyond the period of first transplantation for the less common cancers. Nevertheless, this is the first epidemiological study to examine site-specific cancer incidence after transplant failure. Much larger studies have the potential to further illuminate the relation between risk of specific cancer types and current immune function.

\section{Comparison with other studies}

The reduction in incidence of Kaposi's sarcoma and non-Hodgkin's lymphoma after transplant failure is consistent with a role for current functional immunity in the prevention of these cancers. Both Kaposi's sarcoma and non-Hodgkin's lymphoma are causally associated with infection by $\gamma$ herpesviruses: Kaposi's sarcoma with human herpesvirus-8, and non-Hodgkin's lymphoma with Epstein-Barr virus in a large proportion of immunodeficient cases. ${ }^{22}$ For both cancers, reduction in immunosuppression allows reconstitution of control by herpesvirus-specific CD8 T cells. ${ }^{2324}$ Consistent with the pattern seen in HIV infection, ${ }^{23}$ our analyses suggest that restoration of immune function can prevent these cancers from occurring, even in individuals with past intense immunodeficiency. A similar pattern of rapid reduction in risk was not seen for other infection related cancers, although rates were non-significantly lower during dialysis for cancers related to human papillomavirus. Rates of human papillomavirus related cancers decline little, if at all, in the immune reconstitution associated with antiretroviral therapy in people infected with $\mathrm{HIV} \cdot{ }^{4-6}$

Lip cancer and melanoma occur at increased rates after solid organ transplantation and in HIV infection. ${ }^{1}$ The decline in risk of lip cancer on reinstitution of dialysis was of a similar magnitude to that seen for Kaposi's sarcoma and non-Hodgkin's lymphoma. The precise cause of increased risk of lip cancer in transplant recipients remains unclear, although human papillomavirus may play a role ${ }^{25}$ as may individual immunosuppressive agents. ${ }^{13}$ The marked reduction in incidence of cutaneous melanoma observed on reinstitution of dialysis accords with a single previous case report of clinical and dermatoscopic fading of melanocytic naevi after transplant failure and removal of immunosuppression. ${ }^{26}$ Melanoma has no known infectious cause, but there is reliable epidemiological and biological evidence of a relation with immune function. ${ }^{15}$

Rates of thyroid cancer are increased in patients with end stage kidney disease, both before and during dialysis, and after kidney transplantation, ${ }^{18}$ and are not increased in HIV infection. ${ }^{1}$ In our cohort, risk of thyroid cancer was six times higher during dialysis after transplant failure. The higher risk observed during dialysis may reflect the metabolic consequences of chronic renal failure, such as selenium deficiency, ${ }^{27}$ which has been linked with thyroid cancer risk in one nested case-control study. ${ }^{28}$ Case reports of thyroid 


\section{WHAT IS ALREADY KNOWN ON THIS TOPIC}

Immunosuppression in solid organ transplant recipients is associated with increased risk of a broad range of cancers

The effect on cancer risk of reduction or cessation of such immunosuppression is largely unknown

\section{WHAT THIS STUDY ADDS}

Increased cancer risk is rapidly reversible on reduction of immunosuppression after kidney transplant failure for some, but not all, cancer types

For Kaposi's sarcoma, non-Hodgkin's lymphoma, melanoma, and lip cancer, the oncogenic effect of immunosuppression was rapidly reversed when immunosuppression ceased

For leukaemia, lung cancer, and cancers related to end stage kidney disease, however, risk remained significantly elevated after transplant failure

cancer occurring in individuals with secondary hyperparathyroidism induced by hypocalcaemia have also been described. ${ }^{29}$

Rates of leukaemia, and cancers of the stomach, lung, and colon are increased after kidney transplantation ${ }^{18}$ and, except for colon cancer, during $\mathrm{HIV}$ infection. ${ }^{1}$ Risk of these cancers was not associated with currency of immunosuppression in this study; this could suggest that they are not closely related to current immune function or may simply reflect a lack of statistical power. Rates of breast and prostate cancer, which are not increased either after solid organ transplantation or in HIV infection, ${ }^{1}$ were not increased at any stage and showed no particular pattern with respect to currency of immunosuppression.

\section{Conclusions and policy implications}

The rapid reduction of risk on cessation of immunosuppression for certain viral and immunodeficiency related cancers demonstrates that the effect of immunosuppression is largely reversible for these cancers. An opposite pattern of increased risk during dialysis was observed for thyroid cancer, suggesting that a factor related to end stage kidney disease is important in its pathogenesis. These findings may help inform the management of cancer risk after solid organ transplantation, as well as in HIV infection, where improvements in immune function from new treatments are changing the spectrum of cancer risk. They also offer novel insight into the role of current functional immunity in the prevention of cancer in the general population.

We acknowledge the dedication and care with which dialysis and transplantation units throughout Australia have regularly submitted the information on which this analysis has been performed, and the ANZDATA Registry staff who have created and maintained the database so accurately. The interpretation and reporting of these data are the responsibility of the authors and in no way should be seen as official policy or interpretation of the Australia and New Zealand Dialysis and Transplant Registry. We thank the staff of the state and territory cancer registries for the use of their data. We also acknowledge assistance by the Australian Institute of Health and Welfare, and the Cancer Council Victoria, in the conduct of this study.

Author contribution: All authors contributed to the research design. Data were collected by the ANZDATA Registry and the Australian Association of Cancer Registries. Statistical analysis was performed by MTVL and JA.
All authors contributed to the analysis and interpretation of data. MTVL, CMV, and AEG were responsible for drafting the manuscript. All authors have contributed to revisions and reviewed the final manuscript. Funding and disclosures: This work was supported by the Cancer Council New South Wales (RG 47/03); the National Health and Medical Research Council (ID 510346 to CMV, ID 401131 to MTVL); and the Cancer Institute New South Wales (07/CDF/1-38 to CMV, 06/RSA/1/28 to MTVL). The ANZDATA Registry administrative office is supported by funding from the Australian Government Department of Health and Ageing, the New Zealand Ministry of Health, and Kidney Health Australia; data collection costs were borne by contributing renal units. The National Centre in HIV Epidemiology and Clinical Research is funded by the Australian Government Department of Health and Ageing and is affiliated with the Faculty of Medicine, University of New South Wales. The funders had no role in the design and conduct of the study; collection, management, analysis, and interpretation of the data; or preparation, review, or approval of the manuscript.

Competing interests: JRC is on advisory boards and speaker panels for pharmaceutical companies Astellas, Novartis, Wyeth, and Hoffmann la Roche, and he has received research support from the Juvenile Diabetes Foundation International, Novartis, Wyeth, Jannsen-Cilag, and Hoffmann la Roche. AEG is on the advisory board for the Gardasil human papillomavirus vaccine for the Commonwealth Serum Laboratories. No other authors reported financial disclosures.

Ethical approval: Approval was granted by all relevant institutional ethics committees. The requirement for informed consent from patients was waived because the researchers received only de-identified data. Data sharing: No additional data available.

1 Grulich AE, van Leeuwen MT, Falster MO, Vajdic CM. Incidence of cancers in people with HIV/AIDS compared with immunosuppressed transplant recipients: a meta-analysis. Lancet 2007:370:59-67.

2 Franceschi S, Maso LD, Rickenbach M, Polesel J, Hirschel B, Cavassini M, et al. Kaposi sarcoma incidence in the Swiss HIV Cohort Study before and after highly active antiretroviral therapy. Br J Cancer 2008;99:800-4.

3 Polesel J, Clifford GM, Rickenbach M, Dal Maso L, Battegay M, Bouchardy C, et al. Non-Hodgkin lymphoma incidence in the Swiss HIV Cohort Study before and after highly active antiretroviral therapy. AIDS 2008;22:301-6.

4 Clifford GM, Polesel I, Rickenbach M, Dal Maso L, Keiser O, Kofler A et al, for the Swiss HIV Cohort. Cancer risk in the Swiss HIV Cohort Study: associations with immunodeficiency, smoking, and highly active antiretroviral therapy. I Natl Cancer Inst 2005;97:425-32.

5 Patel P, Hanson DL, Sullivan PS, Novak RM, Moorman AC, Tong TC, et al. Incidence of types of cancer among HIV-infected persons compared with the general population in the United States, 19922003. Ann Intern Med 2008;148:728-36.

6 Hessol NA, Pipkin S, Schwarcz S, Cress RD, Bacchetti P, Scheer S. The impact of highly active antiretroviral therapy on non-AIDS-defining cancers among adults with AIDS. Am I Epidemiol 2007;165:1143-53.

7 Penn I. Depressed immunity and the development of cancer. Cancer Detect Prev 1994:18:241-52.

8 Lebbé C, Legendre C, Francès C. Kaposi sarcoma in transplantation. Transplant Rev 2008;22:252-61.

9 Starzl TE, Nalesnik MA, Porter KA, Ho M, Iwatsuki S, Griffith BP, et al. Reversibility of lymphomas and lymphoproliferative lesions developing under cyclosporin-steroid therapy. Lancet 1984;1:583-7.

10 Penn I. The role of immunosuppression in lymphoma formation. Springer Semin Immunopathol 1998;20:343-55.

11 Marcen R, Teruel JL. Patient outcomes after kidney allograft loss. Transplant Rev 2008;22:62-72.

12 Hill AB. The environment and disease: association or causation? Proc $R$ Soc Med 1965;58:295-300.

13 van Leeuwen MT, Grulich AE, McDonald SP, McCredie MRE, Amin J, Stewart JH, et al. Immunosuppression and other risk factors for lip cancer after kidney transplantation. Cancer Epidemiol Biomarkers Prev 2009;18:561-9.

14 van Leeuwen MT, Grulich AE, Webster AC, McCredie MRE, Stewart JH, McDonald SP, et al. Immunosuppression and other risk factors for early and late non-Hodgkin lymphoma after kidney transplantation. Blood 2009;114:630-7.

15 Vajdic CM, van Leeuwen MT, Webster AC, McCredie MRE, Stewart JH, Chapman IR, et al. Cutaneous melanoma is related to immune suppression in kidney transplant recipients. Cancer Epidemiol Biomarkers Prev 2009;18:2297-303.

16 McDonald SP, Russ GR, Kerr PG, Collins JF. ESRD in Australia and New Zealand at the end of the millennium: a report from the ANZDATA registry. Am J Kidney Dis 2002;40:1122-31. 
17 Australian Institute of Health and Welfare (AlHW) and Australasian Association of Cancer Registries (AACR). Cancer in Australia: an overview, 2008. AlHW, 2008.

18 Vajdic CM, McDonald SP, McCredie MR, van Leeuwen MT, Stewart JH, Law $M$, et al. Cancer incidence before and after kidney transplantation. JAMA 2006;296:2823-31.

19 Breslow NE, Day NE. Statistical methods in cancer research. Vol IIthe design and analysis of cohort studies. Oxford University Press, 1987.

20 Hirji KF, Tsiatis AA, Mehta CR. Median unbiased estimation for binary data. Am Stat 1989;43:7-11.

21 Bouvard V, Baan R, Straif K, Grosse Y, Secretan B, Ghissassi FE, et al. A review of human carcinogens-part B: biological agents. Lancet Oncol 2009;10:321-2.

22 International Agency for Research on Cancer (IARC). IARC monographs on the evaluation of carcinogenic risks to humans: Epstein-Barr virus and Kaposi's sarcoma Herpes virus/Human Herpes virus 8. IARC Press, 1997.

23 Young L, Alfieri C, Hennessy K, Evans H, O’Hara C, Anderson KC, et al. Expression of Epstein-Barr virus transformation-associated genes in tissues of patients with EBV lymphoproliferative disease. N Engl J Med 1989;321:1080-5.
24 Barozzi P, Bonini C, Potenza L, Masetti M, Cappelli G, Gruarin P, et al. Changes in the immune responses against human herpesvirus-8 in the disease course of posttransplant Kaposi sarcoma. Transplantation 2008;86:738-44.

25 International Agency for Research on Cancer. IARC monographs on the evaluation of carcinogenic risks to humans: human papillomaviruses. IARC Press, 2007.

26 Piaserico S, Alaibac M, Fortina AB, Peserico A. Clinical and dermatoscopic fading of post-transplant eruptive melanocytic nevi after suspension of immunosuppressive therapy. J Am Acad Dermatol 2006;54:338-40.

27 Vamvakas S, Bahner U, Heidland A. Cancer in end-stage renal disease: potential factors involved. Am I Nephrol 1998;18:89-95.

28 Glattre E, Thomassen Y, Thoresen SO, Haldorsen T, Lund-Larsen PG, Theodorsen L, et al. Prediagnostic serum selenium in a case-contro study of thyroid cancer. Int J Epidemiol 1989;18:45-9.

29 Klyachkin ML, Sloan DA. Secondary hyperparathyroidism: evidence for an association with papillary thyroid cancer. Am Surg 2001;67:397-9.

Accepted: 23 October 2009 